\title{
DISCUTINDO SOBRE ESTRESSE E ENFRENTAMENTO DA PREMATURIDADE POR CUIDADORES
}

\author{
DISCUTINDO SOBRE ESTRÉS Y ENFRENTAMIENTO DE LA \\ PREMATURIDAD POR CUIDADORES
}

\section{DISCUSSING ABOUT STRESS AND COPING WITH THE PREMATURITY BY CAREGIVERS}

\author{
Ana Cristina Barros da CUNHA ${ }^{1}$ \\ Julie Anna Barros SMITH ${ }^{2}$ \\ Laila Pires Ferreira AKERMAN ${ }^{3}$ \\ Vanessa Oliveira de SOUZA ${ }^{4}$
}

RESUMO: Baseado em delineamento descritivo exploratório, nosso objetivo foi discutir sobre o enfrentamento da prematuridade com base na investigação de relações entre estresse materno, estratégias de enfrentamento e variáveis sociodemográficas de mães de crianças entre 0 e 3 anos, atendidas no serviço de follow-up da Maternidade escola da UFRJ. Todas responderam a Escala Modos de Enfrentamento do Problema e o Inventário de Sinais e Sintomas de Stress. Correlações estatísticas significativas não foram encontradas entre as variáveis estudadas; entretanto, $57.2 \%$ apresentaram estresse em diferentes níveis. A estratégia de enfrentamento mais prevalente foi o "foco no problema" para mães com e sem sintomas de estresse. Conclui-se que esse tipo de estratégia seria a mais comum no enfrentamento da prematuridade, porque ajuda a mãe na sua preocupação primária com a condição médica do filho.

PALAVRAS-CHAVE: Enfrentamento. Estresse. Prematuridade. Cuidadores.

RESUMEN: De acuerdo con el diseño descriptivo exploratorio, nuestro objetivo fue discutir sobre el enfrentamiento de la prematuridad con base en la investigación de relaciones entre estrés materno, estrategias de enfrentamiento y variables sociodemográficas de madres de niños entre 0 y 3 años, atendidas en el servicio de seguimiento de la Maternidad escuela de la UFRJ. Todas respondieron a la Escala Modos de Enfrentamiento del Problema y el Inventario de Señales y Síntomas de Tensión. Las correlaciones estadísticas significativas no se encontraron entre las variables estudiadas; sin embargo, el $57.2 \%$ presentó estrés en diferentes niveles. La estrategia de enfrentamiento más prevalente fue el "foco en el problema" para madres con y sin síntomas de estrés. Se concluye que ese tipo de estrategia sería la más común

${ }^{1}$ Universidade Federal do Rio de Janeiro (UFRJ), Rio de Janeiro - RJ - Brasil. Instituto de Psicologia, Maternidade escola - UFRJ. E-mail: acbcunha@yahoo.com.br

${ }^{2}$ Universidade Federal do Rio de Janeiro (UFRJ), Rio de Janeiro - RJ - Brasil. Instituto de Psicologia, Maternidade escola - UFRJ. E-mail: julieannabsmith@ gmail.com

${ }^{3}$ Universidade Federal do Rio de Janeiro (UFRJ), Rio de Janeiro - RJ - Brasil. Instituto de Psicologia, Maternidade escola - UFRJ. E-mail: lailaakerman@ gmail.com

${ }^{4}$ Universidade Federal do Rio de Janeiro (UFRJ), Rio de Janeiro - RJ - Brasil. Instituto de Psicologia, Maternidade escola - UFRJ. E-mail: sane_rj@hotmail.com 
en el enfrentamiento de la prematuridad, porque ayuda a la madre en su preocupación primaria con la condición médica del hijo.

PALABRAS-CLAVE: Enfrentamiento. Estrés. Prematuridad. Cuidadores.

ABSTRACT: Based on an exploratory descriptive design, we aim to discuss the coping face to the preterm birth according to the investigation of relations among maternal stress, coping strategies and socio demographic data of 42 mothers of $0-3$ year-old children, who were attended at the follow-up service of Maternidade escola of Universidade Federal do Rio de Janeiro. All of them answered the Escala Modos de Enfrentamento do Problema (Ways of coping with problems), and the Inventário de Sinais e Sintomas de Stress (Inventory of stress signs and symptoms). Significant statistical correlations among the variables studied were not found. Nevertheless, $57.2 \%$ of caregivers presented different levels of stress. The most prevalent coping strategy was "focus on the problem" used by mothers with or without stress symptoms. We conclude that this type of strategy would be the most common in coping with prematurity, because it helps the mother in her primary concern with the child's medical condition.

KEYWORDS: Coping. Stress. Prematurity. Caregivers.

\section{Introdução}

Nascimentos pré-termos ou prematuros são aqueles que ocorrem antes da $37^{\mathrm{a}}$ semana gestacional, quando frequentemente o recém-nascido $(\mathrm{RN})$ pesa abaixo de $2500 \mathrm{~g}$ e requer cuidados terapêuticos intensivos (FEBRASGO, 2011). A prematuridade é considerada uma questão de saúde pública e alvo de políticas públicas de saúde no Brasil e no mundo (HOWSON; KINNEY; LAWN, 2012; LEAL et. al 2016). Desde o relatório publicado pela Unicef (2013) em parceria com o Ministério da Saúde, a prevalência de nascimentos prematuros no Brasil vem aumentando e atualmente representa 9,2\% dos nascimentos no país (TABILE et al, 2016). Ressalta-se nesta prevalência a inclusão de "prematuros tardios", termo usado para designar o RN nascido entre a $34^{\mathrm{a}}$ e a $36^{\mathrm{a}}$ semanas, cujos índices incrementam as taxas de prematuridade, que se elevam de acordo com o aumento de intervenções obstétricas para redução de complicações maternas e fetais, como, por exemplo, a cesariana (LEAL et al, 2016). Sem evidências suficientes de indicação médica, o Brasil vem sendo reconhecido como um país com as maiores taxas de cesariana no mundo (VICTORIA et al, 2011), com índices alarmantes de 56\% do total de partos em 2013, sendo $43 \%$ nos serviços de saúde pública e $88 \%$ no setor privado (DOMINGUES et al, 2014). 
Paralelamente, o Brasil assiste a uma diminuição nas taxas de mortalidade infantil, o que se relaciona a implementação de programas de atenção à saúde maternoinfantil, como as Unidades de Terapia Intensiva Neonatal (UTIN) e os serviços de followup do desenvolvimento infantil (VICTORIA et al, 2011). Quando o bebê é retirado precocemente do ambiente uterino, suas condições clínicas devem ser constantemente observadas, devido sua imaturidade fisiológica e neurológica, que acarretam prejuízos para o desenvolvimento e a saúde do RN. Esses prejuízos são confirmados pela literatura, que afirma ser a prematuridade um fator de risco com impacto importante para o desenvolvimento infantil(HOWSON et al, 2013; SANTOS et al, 2013). Concomitantemente, existem as dificuldades associadas à internação em UTIN, necessária na maioria das vezes para a sobrevivência do RN prematuro (GASPARDO; MARTINEZ; LINHARES, 2010). Ao mesmo tempo em que as internações em UTIN colaboram para a proteção e cuidado do $\mathrm{RN}$, ela traz repercussões para a construção do vínculo mãe-bebê pela inevitável separação entre RN e seus cuidadores (KORJA; LATYA; LEHTONEN, 2012; ALEXANDRE et al, 2016), assim como para o desenvolvimento emocional infantil, que é afetado pelas dificuldades de se estabelecer um vínculo afetivo de apego entre mãe e bebê devido a uma menor interação no pós-parto imediato, especialmente em situações de internação em UTIN. Para os cuidadores, o nascimento de um filho prematuro resulta em condição de vulnerabilidade psíquica e a internação na UTIN amplia o potencial estressor e desestruturante da prematuridade, o que repercute em estresse para a mãe e afeta toda a família (DADALTO; ROSA, 2015; PONTES; CANTILLINO, 2014).

Compreende-se por estresse um padrão de respostas com componentes físicos e psicológicos, apresentado diante de estímulos ou acontecimentos que perturbam o equilíbrio do organismo e que causa impacto negativo no seu funcionamento global (LIPP, 2004; LIPP; BARGAS, 2013). Lipp (2004) propõe a avaliação e classificação do estresse em quatro níveis, que variam desde uma fase de alerta até a de exaustão. Todas as fases do estresse têm caraterísticas específicas, sendo: 1) fase de alerta: é uma fase positiva do estresse caracterizada pelo aumento de adrenalina, energia e vigor, que prepara a pessoa para enfrentar o evento estressor, mas que resulta em sinais de tensão muscular, irritabilidade, nervosismo, sensibilidade excessiva, ansiedade e inquietação; 2) fase de resistência: é uma fase de tentativa de reequilíbrio resultante do acúmulo de estresse, caracterizada pelo surgimento de dificuldades com a memória e muito cansaço; 3) fase de quase-exaustão: é a fase em que a tensão excede o limite do gerenciável e é 
caracterizada por sintomas como cansaço mental, dificuldade de concentração, perda de memória imediata, apatia, problemas físicos (gastrite ou úlcera, por exemplo), mudanças de apetite, autodúvidas, ansiedade, pressão alta e queda da qualidade de vida; e; 4) fase de exaustão, que é a fase mais negativa do estresse, caracterizada por sintomas como sono desregulado, dificuldades sexuais, incapacidade de trabalhar, desgaste físico, cansaço excessivo e dificuldades sociais. Os níveis de estresse se elevam por meio de sintomas, físicos, psicológicos ou mistos, que se agravam progressivamente ao longo das fases descritas. Isto significa dizer que uma pessoa pode se encontrar em uma fase de alerta do estresse, mas se o evento estressor se intensificar e gerar um acúmulo os sintomas podem aumentar e dará início a uma fase mais grave do estresse.

Diversas questões dão o contorno estressante e de vulnerabilidade psíquica da situação de prematuridade para cuidadores de RN prematuros. Por exemplo, quando a internação na UTIN é mandatória, os cuidadores, principalmente a mãe, se sentem especialmente mobilizados psicologicamente devido à iminência do risco de morte do filho prematuro hospitalizado (GOODING et al., 2011; MOUSQUER et al, 2014). Após a alta hospitalar, suprir as demandas do bebê prematuro, antes delegados à equipe de saúde, também pode resultar em estresse para os cuidadores, sobretudo para aqueles que se sentem incapazes e não preparados para lidar com a fragilidade imposta pela prematuridade (DADALTO; ROSA, 2015; FAVARO; PERES; SANTOS, 2012). Dessa forma, a internação na UTIN é um importante estressor e fator de impacto para os cuidadores, que também se sentem "prematuros" (grifo nosso) e desamparados no exercício de sua maternidadelpaternidade (DADALTO; ROSA, 2015). Os cuidadores se deparam com a frustração de suas expectativas iniciais sobre o bebê e o parto, que interrompem precocemente o processo de idealização dos primeiros cuidados ao bebê (OLIVEIRA et al, 2013; PERLIN; OLIVEIRA; GOMES, 2011). O bebê imaginário, "perfeito", construído pela mãe durante a gestação, não corresponde ao bebê real, com seu corpo frágil, pequeno e em condições de saúde que dificultam, muitas vezes, a interação da díade mãe-bebê e a construção de um vínculo afetivo de apego, tão importante para o desenvolvimento futuro (FLECK; PICCININI, 2013; PONTES; CANTILLINO, 2014; BOWLBY, 1989).

Literatura indica ainda que tais cuidadores apresentam sintomas de estresse resultante de questões como o medo de segurar seus bebês, a subordinação aos profissionais de saúde e a preocupação em prover um cuidado mais técnico percebido como o melhor e mais adequado às necessidades do filho (JUBINVILLE et al, 2012). 
Essas questões fazem com que a mãe passe de um estado de "preocupação materna primária", tal como proposto por Winnicott (2000\1956), para uma condição de "preocupação médica primária” (AGMAN et al, 1999), quando ela investe em se capacitar nas questões médicas relacionadas à condição clínica do bebê. Nesse sentido, a prematuridade é potencialmente estressante e dificulta que a mãe exerça plenamente sua maternidade, porque faz com que ela não invista subjetivamente em um estado psicológico que a torna sensível às necessidades básicas de seu filho. Diante dessa condição de tamanha vulnerabilidade psíquica, fatores de proteção como possuir um vínculo empregatício, associado a fatores como estado civil, nível educacional e acompanhamento adequado durante o pré-natal podem minimizar o estresse desses cuidadores (DITZEN; HEINRICHS, 2014). Por outro lado, escassez de recursos materiais, condições de emprego desfavoráveis e complicações no ciclo gravídico puerperal podem potencializar os sintomas de estresse decorrentes da prematuridade (SCHETTER; TANNER, 2012).

No contexto do nascimento prematuro de um filho, não é incomum que os cuidadores apresentem além do estresse, depressão, aumento da ansiedade e sentimentos ambivalentes, como raiva pela separação imposta pela internação na UTIN, culpa pela situação do bebê e gratidão à equipe de saúde pelo trabalho realizado (FAVARO; PERES; SANTOS, 2012; OLIVEIRA et al, 2013; FLECK; PICCININI, 2013; JUBINVILLE et al., 2012; SHAW, 2013). Para lidar com tudo isso, os cuidadores investem em um processo de enfrentamento baseado em estratégias para minimizar as consequências da vulnerabilidade psíquica que a prematuridade impõe. Entende-se por enfrentamento os esforços cognitivos elou comportamentais utilizados para manejar demandas externas e/ou internas específicas que são avaliadas como ameaçadoras e que sobrecarregam ou excedem os recursos pessoais (LAZARUS; FOLKMAN, 1984). Focado no problema, na emoção ou em ambos, o enfrentamento pode reunir diferentes tipos de estratégias. Se voltar para a compreensão racional do problema, buscar suporte social da rede de apoio ou usar a religiosidade como fonte de apoio emocional, por exemplo, podem ser estratégias adotadas simultaneamente para lidar com as demandas de uma situação específica. Independentemente do tipo de estratégia, o enfrentamento se refere a um modo de relação da pessoa com o estressor, cujo objetivo é impedir o desequilíbrio e o descontrole, auxiliando na elaboração psíquica das repercussões de uma condição estressora, como a prematuridade, por exemplo, (ZANETTI; STUMM; UBESSI, 2013). 
Literatura sobre enfrentamento e estresse no contexto da prematuridade tem concluído que o nascimento prematuro tem impacto psicológico significativo, porque resulta em aumento dos níveis de ansiedade e estresse nos cuidadores (FAVARO; PERES; SANTOS, 2012; JUBINVILLE et al, 2012; SHAW, 2013). Assim, as estratégias de enfrentamento devem ajudar os cuidadores a lidarem da melhor forma possível com as demandas da prematuridade a fim deles conseguirem de forma resolutiva manejar o estresse relacionado à vivência do nascimento de um filho prematuro (ZANETTI; STUMM; UBESSI, 2013; RODRIGUES; SIQUEIRA, 2013). Para enfrentar esse estresse, os cuidadores adotam diferentes estratégias de enfrentamento, destacando-se as estratégias de cunho religioso, focalizadas no problema elou na busca de suporte social (SHAW, 2013; ZANETTI; STUMM; UBESSI, 2013; RODRIGUES; SIQUEIRA, 2013). Diante do exposto e considerando a prematuridade como uma situação inesperada que gera vulnerabilidade psíquica com desdobramentos que repercutem em estresse, nosso objetivo foi ampliar a discussão sobre o enfrentamento materno da prematuridade buscando investigar relações entre estresse materno, estratégias de enfrentamento e variáveis sociodemográficas em cuidadoras de crianças nascidas prematuras atendidas em um ambulatório de follow-up do desenvolvimento infantil e que foram internadas em UTIN ao nascer.

\section{Método}

Com base em um delineamento descritivo e exploratório, com caráter quantitativo e transversal, o estudo foi conduzido com 42 cuidadoras (todas mães) de crianças entre zero e três anos de idade atendidas no serviço de followup da Maternidade escola da Universidade Federal do Rio de Janeiro e que ao nascer precisaram ficar internadas na UTIN da instituição pelo prazo mínimo de 48 horas. As cuidadoras foram convidadas a participar da pesquisa durante a espera para consulta médica e, após assinarem um Termo de Consentimento Livre Esclarecido (TCLE) aprovado pelo Comitê de Ética da instituição (CAEE n. 06386412.1.0000.5275), responderam a um Protocolo de dados gerais para coleta de dados sociodemográficos e variáveis psicossociais (idade, escolaridade, estado civil, paridade, vínculo empregatício, religião e suporte social percebido), ao Inventário de Sintomas de Estresse para Adultos de LIPP - ISSL (LIPP, 2000), para identificar níveis de estresse 
classificados em quatro fases (de alerta, resistência, de quase-exaustão e exaustão) e a Escala Modos de Enfrentamentos de problemas - EMEP (SEIDL, TRÓCCOLI, ZANNON, 2001), para identificar as estratégias de enfrentamento adotadas frente ao estressor prematuridade.

O ISSL é um instrumento psicométrico brasileiro de avaliação psicológica que classifica o estresse por meio da soma de sintomas físicos, psicológicos ou mistos típicos de cada uma das fases do estresse já mencionadas (LIPP, 2004). Já a EMEP é uma escala do tipo Likert composta por 45 itens, distribuídos em quatro fatores: 1) enfrentamento focalizado no problema (estratégias voltadas para o manejo e reavaliação do problema), 2) enfrentamento focalizado na emoção (estratégias que envolvem esquiva, negação, pensamento irrealista, atribuição de culpa, afastamento do problema e funções paliativas frente a um estressor); 3) enfrentamento focalizado na busca de práticas religiosas \pensamento fantasioso (estratégicas focadas em pensamentos religiosos e fantasiosos.); e 4) enfrentamento focalizado na busca por suporte social (estratégicas focadas na procura por apoio social e busca de informações). Cabe destacar que a EMEP é uma das poucas, senão a única, escala brasileira criada para avaliação do enfrentamento em contextos de saúde e tem sido usada em estudos com mães de bebês prematuros internados em UTIN (MOTTA-LOSS et al., 2015), além de outras populações.

Os dados do protocolo foram processados em termos de análise de frequência de ocorrência das categorias citadas e os demais dados foram processados de acordo com as normas e instruções dos instrumentos. Todos os dados foram analisados estatisticamente, buscando-se investigar, em termos descritivos e correlacionais, relações entre as principais variáveis de interesse do estudo, estresse, estratégias de enfrentamento e variáveis sociodemográficas (idade, escolaridade, estado civil, paridade, vínculo empregatício e religião). Utilizou-se o programa SPSS (Statistical Package for the Social Sciences) versão 17.0 (SPSS Inc., Chicago, IL, EUA) para análise correlacional entre os dados, adotando-se $p \leq 0,05$ como nível de significância.

\section{Resultados}

A média de idade das cuidadoras foi de 28,8 anos. Sobre a escolaridade, 28,5\% $(n=12)$ delas possuíam ensino fundamental, 57,2\% ( $n=24)$ ensino médio, 12\% ( $n=5)$ 
ensino superior e 1 participante não respondeu. Do total, $70 \%(\mathrm{n}=29)$ eram casadas e $50 \%(\mathrm{n}=21)$ eram primigestas. Mais da metade $(64,2 \% ; \mathrm{n}=27)$ declarou possuir vínculo empregatício em atividades como comércio, serviços domésticos, administração, etc. Referente à religião, $88 \%(\mathrm{n}=37)$ delas declararam professar alguma religião, sendo a maioria católica $(61,9 \% ; n=26)$, seguida de protestantes $(23,8 \% ; n=10)$ e espíritas $(4,76 \% ; n=1)$. Grande parte da amostra $(71,4 \% ; n=30)$ declarou possuir suporte social, relatando principalmente auxílio de familiares na execução de tarefas domésticas.

Em relação ao estresse, mais da metade das cuidadoras $(57,1 \%$; $n=24)$ apresentou sinais e sintomas de estresse em alguma fase e nível de gravidade. Dentre estas, a maior frequência foi de cuidadoras na fase de resistência $(26,1 \%, n=11)$, seguida da fase de quase exaustão $(19 \% ; n=8)$, fase de exaustão $(7,1 \% ; n=3)$ e fase de alerta $(4,7 \% ; n=2)$ do estresse.

Dados comparativos do estresse pelo ISSL e das estratégias de enfrentamento pela EMEP estão apresentados na Figura 1. Comparando as estratégias de enfrentamento mais prevalentes entre as cuidadoras com $(57,2 \%, n=24)$ e sem estresse $(42,8 \%, n=18)$, observa-se que para ambos os grupos o enfrentamento "focalizado no problema" foi o mais usado pelas cuidadoras. No entanto, na segunda estratégia mais prevalente os grupos se diferenciaram. Para as cuidadoras com estresse, as estratégias "focalizadas na busca de práticas religiosas \pensamento fantasioso" foram a segunda mais prevalente apresentada por $29,1 \%(n=07)$; enquanto que essa estratégia foi a terceira mais adotada pelas cuidadoras sem estresse $(16,6 \% ; n=3)$, que usaram como segundo tipo de enfrentamento mais prevalente as estratégias "focalizadas na busca de suporte social" adotadas por $33.3 \%(n=6)$ das cuidadoras sem estresse. Cabe destacar que, a prevalência da estratégia "focalizada na emoção" foi a menor entre as cuidadoras com estresse $(4,7 \%$; n=2) e não foi observada nas mães sem estresse. 
Figura 1: Dados comparativos das estratégias de enfrentamento das cuidadoras com e sem estresse $(\mathrm{N}=42)$

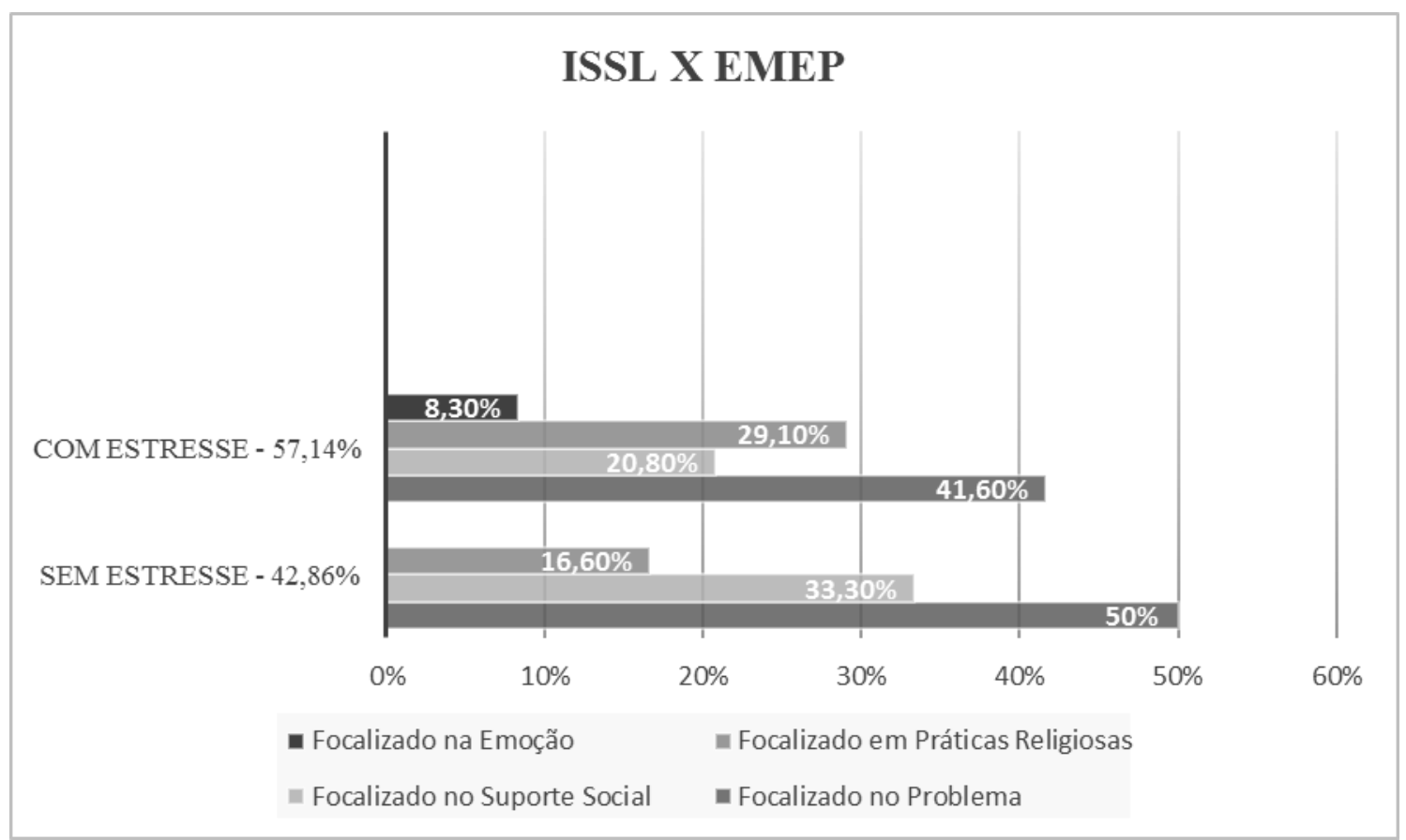

Fonte: Elaboração própria

Correlações de Pearson foram testadas para investigar relações entre níveis de estresse, a estratégia de enfrentamento mais prevalente e as variáveis sociodemográficas (idade, estado civil, escolaridade, paridade, prática de religião e vínculo empregatício) no grupo de cuidadoras com estresse. Observou-se que o estresse não se correlacionou significativamente com a estratégia de enfrentamento $(p=0.70)$ e nem com as variáveis: idade $(p=0.30)$, estado civil $(p=0.92)$, escolaridade $(p=0.45)$, paridade $(p=0.21)$, prática de religião $(p=0.17)$ e vínculo empregatício $(p=0.35)$.

\section{Discussão}

Nossos achados qualificam o contexto do nascimento prematuro como de natureza estressora, visto que 57,2\% das cuidadoras apresentaram estresse em diferentes níveis de gravidade. Tal dado sugere que o parto inesperado e a imprevisibilidade da condição clínica do RN prematuro geram preocupações e frustrações inerentes ao contexto de prematuridade, como temores relacionados à sobrevivência do bebê e às demandas do pós-alta (DADALTO e ROSA, 2015; FAVARO; PERES; SANTOS, 
2012; OLIVEIRA et al, 2013; JUBINVILLE et al, 2012). Diante da maternidade fragmentada pelo nascimento prematuro, tem-se ainda a percepção da mãe de que o RN prematuro não corresponde ao seu ideal de bebê, o que fica agravado pela inviabilidade de vivenciar momentos de interação no pós-parto imediato e experimentar situações esperadas por ela, como a amamentação, o aconchego no colo e o contato físico pele a pele (KORJA et al, 2012; DADALTO; ROSA, 2015; FLECK; PICCININI, 2013).

Por outro lado, não foram encontradas correlações estatisticamente significativas entre o estresse, as estratégias de enfrentamento e nenhuma das variáveis sociodemográficas analisadas (estado civil, paridade, idade, escolaridade, presença de vínculo empregatício e religião), o que pode ter ocorrido devido a limitações amostrais. De todo modo, esses dados parecem confirmar que o contexto da prematuridade tem um potencial de estresse per si, que independe das estratégias de enfrentamento maternas adotadas, assim como de características pessoais ou sociodemográficas que compõem a rede de apoio dos cuidadores. Importante destacar que a grande maioria $(71,4 \%)$ dos cuidadores, incluindo todos os que apresentaram sintomas de estresse, declararam receber suporte social de sua rede de apoio, representado pela ajuda dos familiares na rotina doméstica. Ainda que não tenham sido analisadas correlações estatísticas entre estresse, enfrentamento e suporte social percebido devido a dificuldades amostrais, esse dado para ter relação com o estresse presente em mais da metade da amostra. É sabido que a rede de apoio é fundamental no enfrentamento da prematuridade (MOTTA-LOSS et al, 2015). No entanto, os recursos oferecidos pela rede daqueles cuidadores parecem não ter sido suficientes para minimizar o impacto da prematuridade e prevenir o estresse. O fato de a maior parte da amostra ser casada, já ter filhos ou exercer uma atividade remunerada fora do lar pode, ao invés de serem fatores de proteção, como sugere a literatura (DITZEN; HEINRICHS, 2014), ter representado maior demanda e estresse para aquelas mães.

Dentre as cuidadoras com estresse, a maioria se encontrava na fase de resistência, indicando a ocorrência de um processo de adaptação e de reequilíbrio frente à persistência de um agente estressor. Neste contexto, pode-se pensar no curso da gestação como um período permeado de eventos estressores (SCHETTER; TANNER, 2012), quando o nascimento prematuro potencializaria esse estresse e se associaria a outras fontes de estresse, como as demandas conjugais, familiares e profissionais, haja vista o perfil sociodemográfico das participantes. Para Schetter e Tanner, (2012), falta de suporte financeiro e instrumental e condições de emprego desfavoráveis podem 
potencializar os sintomas de estresse resultantes do nascimento de um filho pré-termo. Estudos qualitativos para investigação da percepção materna sobre sua rede de apoio ou investigações longitudinais para o acompanhamento do estresse e seu enfrentamento durante a gestação até o puerpério poderiam qualificar melhor essa discussão. Outra hipótese para discutir os resultados de estresse seria a de que, tal como afirma a literatura (FLECK; PICCININI, 2013; PONTES; CANTILLINO, 2014), aquelas cuidadoras pautavam seu enfrentamento em uma concepção do filho como um bebê frágil e que necessita de sua atenção e cuidados constantes ao longo de todo o percurso desenvolvimental, especialmente porque passaram por internação em UTIN logo após o nascimento. Isso representaria um impacto emocional que, mesmo a longo prazo pela reverberação daquela concepção de filho frágil, resultaria no estresse materno e poderia se agravar em níveis maiores de estresse, como as fases de quase-exaustão e exaustão apresentadas por $26,1 \%$ das cuidadoras. Literatura afirma que o nascimento prematuro de um filho é uma situação que gera desorganização psíquica e quando a internação na UTIN é compulsória esse estresse é ampliado e tem potencial desestruturante que afeta a dinâmica psíquica da mãe e familiares (DADALTO; ROSA, 2015; PONTES; CANTILLINO, 2014).

Para melhor compreender o enfrentamento desse estresse materno, analisamos os tipos de estratégias adotadas pelas cuidadoras que, possivelmente, contribuíram para um manejo adaptativo à situação de prematuridade. Houve prevalência de estratégias "focalizadas no problema" tanto para as cuidadoras com estresse $(41,60 \%)$ como para aquelas sem estresse (50\%). Isto sugere que, independente da sobrecarga emocional e do estresse que a mãe experimente, a prematuridade exige que ela adote estratégias resolutivas para lidar com a situação. Estratégias "focadas no problema" são resolutivas na medida em que se relacionam com a necessidade materna de obter informações técnicas para compreender melhor a prematuridade do filho e, assim, dar conta da sua “preocupação médica primária". Esse tipo de enfrentamento ajudaria a evitar a desorganização psíquica frente à dor emocional e à possibilidade de morte do bebê (GOODING et al, 2011; MOUSQUER et al., 2014). Ressalta-se ainda que a mãe se depara após a alta da UTIN com a tarefa de suprir, sem a ajuda da equipe médica, as demandas do filho prematuro e isso pode resultar em estresse, agravado quando elas não se sentem capazes de lidar com a fragilidade imposta pela prematuridade (DADALTO; ROSA, 2015; FAVARO; PERES; SANTOS, 2012). 
De todo modo, cabe destacar que esse tipo de enfrentamento pode não ter sido resolutivo e eficaz o suficiente para evitar o estresse próprio desse tipo de situação, já que, mesmo sem terem sido encontradas correlações estatísticas significativas entre os tipos de enfrentamento e os níveis de estresse, mais da metade da amostra apresentou estresse, inclusive com sintomas característicos de fases mais críticas. Isso nos faz questionar o uso da estratégia "focada no problema" como um único recurso adaptativo no contexto da prematuridade. Logicamente, limitações amostrais não permitem a generalização desses dados, que devem ser analisados associados às demais estratégias de enfrentamento adotadas. A estratégia "focalizada no suporte social", por exemplo, a segunda mais prevalente para o grupo de cuidadoras sem estresse, foi apresentada por $33,33 \%$ delas. Isto sugere que para enfrentar o estresse decorrente da prematuridade as cuidadoras combinam estratégias de enfrentamento se utilizando de recursos que os auxiliem a lidar com a prematuridade, tanto através da aquisição de conhecimentos sobre o problema como na busca de auxilio e suporte da sua rede de apoio. Isso confere um caráter multidimensional no processo de enfrentamento da prematuridade, quando devem ser conjugados recursos e estratégias de racionalização como o "foco no problema" com a mobilização de recursos ecológicos, como a "busca de suporte social". Se apropriar de um suporte mais técnico voltado para entender as condições de saúde do filho e as peculiaridades do nascimento prematuro (hospitalização na UTIN, permanência no ambiente hospitalar etc.), assim como lançar mão da rede de apoio e do apoio institucional que oferece suporte técnico e integral aos bebês e suas famílias durante a internação na UTIN como medidas protetoras dos riscos psicológicos podem ajudar a prevenir desfechos negativos para o desenvolvimento infantil e para a saúde materno-infantil.

Importante também discutir o segundo recurso de enfrentamento mais prevalente dentre as cuidadoras com estresse que foram as estratégias "focalizadas na busca de práticas religiosas \pensamento fantasioso". Considerando a alta frequência (88\%) de praticantes de algum tipo de religião dentre as cuidadoras com e sem estresse, sugere-se que o suporte emocional obtido através do exercício da espiritualidade e da prática religiosa pode ter influenciado na resposta emocional de estresse. Estudos sobre estresse e enfrentamento de cuidadores de crianças prematuras já confirmaram que as estratégias de cunho religioso, além das focalizadas no problema elou na busca de suporte social são as mais prevalentes para nesse contexto (SHAW, 2013; ZANETTI; STUMM; UBESSI, 2013; RODRIGUES; SIQUEIRA, 2013). Também usando a EMEP, Seidl 
(2005) constatou que estratégias "focalizadas na busca de práticas religiosas \pensamento fantasioso" costumam ser as mais comumente usadas pelo gênero feminino, característica predominante da amostra estudada.

Não se pode desconsiderar que a ampla presença de uma religião declarada dentre as cuidadoras é compatível com o contexto cultural brasileiro, caracterizado pelo sincretismo religioso. No entanto, na identificação pela EMEP da estratégia "focalizada na busca de práticas religiosas \pensamento fantasioso" também estão incluídos itens que avaliam o uso de pensamento fantasioso para lidar com o estressor. Itens como "Espero que um milagre aconteça" ou "Eu sonho ou imagino um tempo melhor do que aquele em que estou" estão incluídos nesse fator da EMEP e representam um enfrentamento não resolutivo com base no comportamento de esquiva, por exemplo. Para melhor esclarecer esse dado, sugere-se para futuros estudos uma análise de item da EMEP a fim de compreender se para cuidadoras que adotam esse tipo de estratégia os itens de maior pontuação foram aqueles relacionados ao uso do pensamento fantasioso. Não podemos esquecer de que o enfrentamento religioso inclui estratégias (comportamentos, emoções e cognições) que exercem a função de busca de significado e conforto espiritual para situações de vulnerabilidade, se configurando, assim, como uma estratégia adaptativa de busca de conforto, controle e encorajamento, tal como a literatura na área indica (CARTER et al, 2012; KOENIG, 2008).

Ressalta-se então que o enfrentamento da prematuridade se dá como um processo (SHAW et al, 2013) e os diversos tipos de enfrentamentos observados em nosso estudo são esforços empreendidos pelas mães frente uma mesma situação estressora: a prematuridade dos filhos. Logo, é possível que a prevalência das estratégias de enfrentamento "focalizadas no problema", "focalizadas no suporte social" e "focalizadas na prática religiosalpensamento fantasioso" para aquelas cuidadoras estejam relacionadas às características próprias do evento da prematuridade e do seu contexto sociocultural. Buscar conhecer o problema a ser enfrentado, a prematuridade, permitiria elas se preparem para atender a demanda técnica por cuidados especializados e de alta complexidade que o RN prematuro requer. Por sua vez buscar apoio na sua rede de apoio ou no exercício da espiritualidade pode promover o conforto e o suporte emocional necessário para um melhor manejo do estresse próprio dessa situação.

Nossos achados confirmam que a prematuridade, por ser um evento complexo, sobretudo no que se refere ao manejo técnico desconhecido pelos pais, é interpretada pelos cuidadores como um evento estressor que exige atitude resolutiva. Essa 
resolutividade tanto pode se dar de forma racional (com foco no problema) como de forma pragmática com uso de estratégias de busca ativa de apoio (foco na busca de suporte social ou prática religiosa). O tipo de enfrentamento é escolhido como uma resposta às demandas características do contexto de nascimento prematuro, independente da mãe estar estressada ou não, mas considerando as peculiaridades de cada contexto de nascimento prematuro. A escolha das estratégias de enfrentamento decorre da necessidade da mãe reunir esforços e recursos para lidar com esse momento cercado de cuidados especializados e de alta complexidade para seu bebê.

$\mathrm{Na}$ tentativa de controlar a situação do nascimento prematuro inesperado que fragmenta sua maternidade, frequentemente as mães de bebês prematuros precisam adquirir conhecimentos para aprender mais sobre a prematuridade e o contexto técnico da UTIN (DADALTO; ROSA, 2015; OLIVEIRA et al, 2013; PONTES; CANTILLINO, 2014). Dessa forma, elas acreditam poder enfrentar melhor a sua própria vulnerabilidade psíquica permeada pela angústia de ter um filho prematuro (DADALTO; ROSA, 2015). Nesse sentido, ressaltamos a importância do estabelecimento de um laço afetivo de confiança e acolhimento entre profissional de saúde e a mãe "prematura", frustrada pela interrupção prematura do seu processo de maternidade (PERLIN; OLIVEIRA; GOMES, 2011). Especificidades da prematuridade refletem nestas cuidadoras e têm repercussões psicológicas importantes, que podem ser minimizadas quando existe um contato mais estreito e acolhedor com os profissionais de saúde. Eles podem auxiliar na superação do desamparo, angústias e preocupações maternas e no acolhimento das dúvidas, que aumentam a vulnerabilidade psíquica da mãe do RN prematuro (DADALTO \& ROSA, 2015; PONTES; CANTILLINO, 2014; OLIVEIRA et al, 2013). Considerando a frequente necessidade de internação em UTIN, os profissionais de saúde passam a ser figuras de referência para os cuidadores neste ambiente e constantemente são procurados como fonte de suporte informacional e emocional. Dessa forma, eles ajudam os cuidadores a empreenderem um enfrentamento mais adaptativo frente às imprevisibilidades da prematuridade.

\section{Considerações finais}

Conclui-se que o processo de enfrentamento da prematuridade terá sempre a função de auxiliar a lidar com a vulnerabilidade física e psíquica que a prematuridade 
impõe para todos os envolvidos, o que pode ser facilitado quando são oferecidas ações de apoio multiprofissional que ajude nesse enfrentamento e minimize desfechos psicológicos negativos como o estresse. Visitas dos avôs e irmãos do bebê prematuro internado em UTIN, estabelecidos como serviços de rotina na instituição pesquisada, podem ter tido impacto positivo na saúde mental materna, refletindo na ausência de estresse por parte da amostra. Tais ações de atenção à saúde materno-infantil possibilitam para a mãe vivenciar a situação de internação do bebê na UTIN junto com sua rede de apoio, sem um completo afastamento dos familiares. Nesse tipo de ação, todos da rede de apoio adquirem conhecimentos sobre os cuidados do bebê para o pósalta, o que, consequentemente, pode diminuir o impacto do estresse na dinâmica familiar. Além disso, a inserção de familiares e amigos nos cuidados com a criança representa um apoio à mãe e à família, ambos prematuros.

\section{REFERÊNCIAS}

AGMAN, M.; DRUON, C.; FRICHET, A. (1999). Intervenções psicológicas em neonatologia. In: Wanderley, D. B. (Ed.). Agora eu era o rei: os entraves da prematuridade. Salvador: Ágalma, p. 17-34, 1999.

ALEXANDRE, J. D.; MONTEIRO, L.; BRANCO, I.; FRANCO, C. A prematuridade na perspectiva de mães primíparas e multíparas. Análise do seu estado psicoemocional, autoestima e bonding. Análise Psicológica, Lisboa, set. 2016.

BOWLBY, J. Uma base segura: aplicações clínicas da teoria do apego. Ed. Artes Médicas, Porto Alegre: 1989.

CARTER, E. C.; MCCULLOUHG, E.; CARVER, C. S. The mediating role of monitoring in the association of religion with self-control. Social Psycological and Personality Science, mar. 2012.

DADALTO, E. C. V.; ROSA, E.M. Vivências e Expectativas de Mães com Recémnascidos Pré-termo Internados em Unidades de Terapia Intensiva Neonatal. Estudos e Pesquisas em Psicologia, Rio de Janeiro, nov, 2015.

DITZEN, B.; HEINRICHS, M. Psychobiology of social support: The social dimension of stress buffering. Restor Neurol Neurosci, 2014.

DOMINGUES, R. M. et al. Process of decision-making regarding the mode of birth in Brazil: from the initial preference of women to the final mode of birth. Cadernos de saúde publica. 2014, 30 Suppl 1: S1-16. 
FAVARO, M. S. F.; PERES, R. S.; SANTOS, M. A. S. Avaliação do impacto da prematuridade na saúde mental de puérperas. Psico-USF, Bragança Paulista, 2012.

FEBRASGO. Comissões Nacionais Especializadas Ginecologia e Obstetrícia. Manual de orientação: gestação de alto risco. FEBRASGO, Rio de Janeiro, 2011.

FLECK, A.; PICCININI, C. A. O bebê imaginário e o bebê real no contexto da prematuridade, do nascimento ao $3^{\circ}$ mês após a alta. Aletheia, abr. 2013.

GASPARDO, C. M.; MARTINEZ, F. E.; LINHARES, M. B. M. Cuidado ao desenvolvimento: intervenções de proteção ao desenvolvimento inicial de recémnascidos pré-termo. Revista paulista de pediatria, São Paulo, mar. 2010.

GOODING, J. S; COOPER, L. Z.; BLAINE, A. I.; FRANCK, L. S.; HOWSE, J. L.; BERNS, S. D. Family support and family-centered care in the neonatal intensive care unit: Origins, advances, impact. Seminars in Perinatology, fev. 2011.

HOWSON, C. P; KINNEY, M. V.; LAWN, J. E. Born Too Soon: The Global Action Report on Preterm Birth. WHO, New York, 2 mai, 2012.

HOWSON, C. P; KINNEY, M. V.; MCDOUGALL, L; LAWN, J. E. Born too soon: Preterm birth matters. Reprod Health, 2013.

JUBINVILLE, J.; NEBURN-COOK, C.; HEGADOREN, K.; LACAZEMASMONTEIL, T. Symptoms of acute stress disorder in mothers of premature infants. Advances in Neonatal Care, ago. 2012.

KOENIG, H. G. Medicine, religion and health: where Science and spirituality meet. Pennsylvania: Templeton Foundation Press, 2008.

KORJA, R.; LATYA, R.; LEHTONEN, L. The effects of preterm birth on motherinfant interaction and attachment during the infant's first two years. ACTA Obstetricia et Gynecologica Scandinavica, fev. 2012.

LAZARUS, R. S.; FOLKMAN, S. Stress, appraisal and coping. New York: Springer Publishing Company, 1984.

LEAL, M. C.; et al. Provider-Initiated Late Preterm Births in Brazil: Differences between Public and Private Health Services. PLOS ONE 11 (5):2016.

LIPP, M. E. N.; BARGAS, J. A. Estresse e estilo parental materno no transtorno de déficit de atenção e hiperatividade. Psicologia escolar e educacional, 2013.

LIPP, M. E. N. Manual do Inventário de Sintomas de Stress para Adultos de Lipp (ISSL). São Paulo: Casa do Psicólogo, 2000.

MOTTA-LOSS, A. B.; CAPRINI, F. R.; RIGONI, P. V. M. S.; ANDRADE, B. L. S. Estados emocionais e estratégias de enfrentamento de mães de recém-nascidos de risco. Gerais: Revista Interinstitucional de Psicologia, 2015. 
MOUSQUER, P. N; LEÃO, L. C. S.; KEPLER, D. F.; PICCININI, C. A.; LOPES, R. C. S. Mãe, cadê o bebê? Repercussões do nascimento prematuro de um irmão. Estudos de Psicologia, out, 2014.

OLIVEIRA, K.; VERONEZ, M.; HIGARASHI, I. H; CORREA, A. M. C. Vivências de familiares no processo de nascimento e internação dos seus filhos em UTI neonatal. Escola Anna Nery, Rio de Janeiro, jan, 2013.

PERLIN, D. A.; OLIVEIRA, S. M.; GOMES, G. C. A criança na unidade de terapia intensiva neonatal: Impacto da primeira visita da mãe. Revista Gaúcha de Enfermagem, Porto Alegre, set. 2011.

PONTES, G. A. R.; CANTILLINO, A. A influência do nascimento prematuro no vínculo mãe-bebê. Jornal Brasileiro de Psiquiatria, 2014.

RODRIGUES, S. R.; SIQUEIRA, F. J. Suporte familiar e estratégias de enfrentamento de mães com filhos recém-nascidos prematuros internados em unidade de terapia intensiva. Fundação de ensino e pesquisa de Itajubá, 2013.

SANTOS, E. S. L.; KIEVIET, J. F.; ELBURG, R. M.; OOSTERLANN, J. Predictive value of the Bayley Scales of Infant Development on development of very preterm/very low birth weight children: a meta-analysis. Early Human Development, jul. 2013.

SCHETTER, C. D.; TANNER, L. Anxiety, depression and stress in pregnancy: implications for mothers, children, research and practice. Curr Opin Psychiatry, mar. 2012.

SEIDL, E. M. F Enfrentamento, aspectos clínicos e sócio demográficos de pessoas vivendo com HIV/AIDS. Psicologia em Estudo, 2005.

SEIDL, E. M. F.; TRÓCCOLI, B. T.; ZANNON, C. M. L. C. Análise fatorial de uma medida de estratégias de enfrentamento. Psicologia: Teoria e Pesquisa, 2001.

SHAW, R. .; BERNARD, R. S.; STORFER-ISSER, A.; RHINE, W.; HORWITZ, S. Parental Coping in the Neonatal Intensive Care Unit. J Clin Psychol Med Settings, jun, 2013.

TABILE, P. M. et al. Características dos partos pré-termo em hospital de ensino do interior do Sul do Brasil: análise de 6 anos. Revista da AMRIGS, Porto Alegre, jul. 2016.

UNICEF BRASIL. Pesquisa para estimar a prevalência de nascimentos pré-termo no Brasil e explorar possíveis causas. Unicef.org, jul. 2013.

VICTORIA, C. G. et al. Saúde de mães e crianças no Brasil: progressos e desafios. Séries saúde no Brasil, 2011.

ZANETTI, T. G.; STUMM, E. M. F.; UBESSI, L. D. Stress and coping in families of patients in an intensive care unit. Revista de pesquisa cuidado é fundamental, 2013. 


\section{Como referenciar este artigo}

CUNHA, Ana Cristina Barros da, et al. Discutindo sobre estresse e enfrentamento da prematuridade por cuidadores. Temas em Educ. e Saúde, Araraquara, v.13, n.1, p. 4158, jan./jun. 2017. DOI <https://doi.org/10.26673/rtes.v13.n1.jan-jun2017.4.9605>. ISSN: $1517-7947$.

Submetido em: 26/03/2017

Aprovado em: 20/07/2017 